\title{
Lazy, stupid, worthless: A critical commentary of weight bias in healthcare
}

Julie E. Rochefort

\author{
Ryerson University
}

When you think about 'fat' individuals (adults with a BMI of $25 \mathrm{~kg} / \mathrm{m}^{2}$ or greater) what are some of the words that first come to your mind? Lazy? Stupid? Worthless? While this question may seem harsh and perhaps even uncomfortable to answer, a systematic literature review investigating the occurrence of weight bias and stigma among healthcare professionals revealed that physicians, nurses, dietitians and fitness professionals often attribute negative qualities towards overweight and obese individuals. ${ }^{1}$ Common attributions expressed included "non-compliant", "lack of motivation/lazy", and "lack of self-control/self-indulgent". ${ }^{2}$ These beliefs support a view that overeating and sedentary behaviour causes individuals to be or become overweight or obese.

Accordingly, the 2006 Canadian Clinical Practice Guidelines on the management and prevention of obesity prescribes a "lifestyle medication program" aimed to promote weight loss, to decrease individuals' body weight by reducing daily energy intake by $500-1000$ calories and increasing energy expenditure by engaging in at least 30 minutes of moderate physical activity. ${ }^{3}$ While promoting physical activity and healthy eating may not seem like dangerous prescriptions, the clinical focus on weight as an indicator of 'satisfactory progress' has been suggested to underlie the pervasiveness of weight bias within health care. ${ }^{1}$ Despite advances in research since its publication, the clinical practice guidelines are the most current set of recommendations geared towards the management and prevention of obesity in Canada.

Weight-based stereotypes within the medical field have been longstanding ${ }^{5}$ and recent studies have begun to demonstrate the negative impact of such stereotypes on health outcomes. For instance, weight-based discrimination was shown to be positively associated with depression, body image disturbances, and negatively associated with self-esteem. ${ }^{1,4,6}$ Similarly, weight-centered (weight loss) interventions were shown to contribute to body dissatisfactions, disordered eating, weight cycling, and avoidance of health prevention screening and exams due to fear of humiliation, distrust, and unsolicited advice regarding weight loss. ${ }^{1,4,6}$

Some researchers $4,6,7,8$ have argued that the war on obesity is actually a war on obese individuals and the relationship between health and weight may not be as significant as we have been led to believe. For instance, a cross-sectional study of over 5000 American adults revealed that $51 \%$ of overweight individuals and $31 \%$ of obese individuals showed normal cardiometabolic indicators such as elevated blood pressure, triglycerides, cholesterol (HDL), and glucose, as well as insulin resistance and systemic inflammation. ${ }^{9}$ Conversely within the same sample, $23.5 \%$ of normal weight individuals (defined by a BMI $18.5-24.9 \mathrm{~kg} / \mathrm{m}^{2}$ ) had abnormal cardiometabolic indicators. While similar evaluations are needed in Canada, metabolic fitness among overweight and obese individuals may help to explain why a longitudinal study of Canadian adults showed a lower risk of death among overweight individuals compared to normal weight individuals. ${ }^{10}$ While we cannot ignore that many individuals, regardless of their weight continue to demonstrate metabolic abnormalities, research has demonstrated that obese individuals can improve metabolic indicators independent of weight loss ${ }^{11}$

A recently published review paper evaluated the evidence of a weight-neutral practice called Health at Every Size ${ }^{S M}$ (HAES). ${ }^{4}$ The review included six randomized control trials (RCT) comparing a HAES group to a control or diet group. This alternative practice challenges the mentality that weight equates to health and encourages practitioners and patients to shift their focus away from weight. ${ }^{4}$ According to the principal tenets of HAES, individuals are instead, encouraged to respect their natural body shape and size while relying on their hunger and satiety rather than 
c diet, and are guided in active embodiment rather than rigid exercise regimes. ${ }^{4}$ Active embodiment encourages individuals to incorporate joyful body movement into their daily routine as a means to experience the physical and psychological benefits independent of weight loss. While there are limited published HAES studies, the current evidence demonstrates a potential mechanism to overcome weight bias within health care whilst improving the health and well-being of individuals with BMI above established "normal" cut offs, independently of weight loss.

All six RCT HAES groups improved not only their metabolic indicators (e.g. blood pressure, blood lipids) but also, their physical activity levels and eating disorder pathology. Most noteworthy, positive changes in mood, self-esteem and body image were observed. Furthermore, compared to the control group (i.e. weight-loss centered approach), the retention rates were substantially higher in the HAES group. ${ }^{4}$ In one study, the attrition rate was five times higher (42\%) in the diet group versus the HAES (5\%) group ${ }^{4}$ demonstrating a potential shift in patient-centered care.

Despite years of research demonstrating that weightcentered approaches result in poorer outcomes and negative health consequences, ${ }^{4}$ the continued focus on weight within healthcare continues and thereby, raises questions about the providers' primary ethical responsibility: to do no harm. While the prevalence of chronic illnesses among overweight and obese individuals cannot be ignored; using weight loss as a primary health indicator lacks scientific certainty and perpetuates weight-bias within health care.

The studies reviewed here provide a concerning picture about the ubiquity of weight bias among a wide range of health care providers. Weight bias, when present, is unethical and harmful to those health practitioners who purport to treat. It has been suggested that in order to maintain their ethical obligations, healthcare professionals should incorporate a compassionate and weight-neutral approach to their practice. However, in order to overcome weight bias within practice, the first step is to recognize and acknowledge bias. So, when you think about 'fat' individuals what are some of the words that first come to your mind?

\section{References}

1. Puhl R, Heuer C. The Stigma of Obesity: A Review and Update. Obesity. 2009; 17(5):941-964.

2. Schwartz MB. Weight Bias among Health Professionals Specializing in Obesity. Obesity Research. 2003;11(9):1033-1039.

3. Lau DCW, Douketis JD, Morrison KM, et al. Canadian clinical practice guidelines on the management and prevention of obesity in adults and children. Canadian Medical Association Journal. 2006;176 (8):1-117.

4. Bacon L, Aphramor L. (2011). Weight Science: Evaluating the Evidence for a Paradigm Shift. Nutrition Journal. 2011;10(9). Available from: http://www. nutritionj.com/content/pdf/1475-2891-10-9.pdf

5. Price JH, Desmond SM, Krol RA. et al. (1987). Family Practice Physicians' Beliefs, Attitudes and Practices Regarding Obesity. American Journal of Preventative Medicine. 1987;3:339-345

6. Neumark-Sztainer D. Preventing obesity and eating disorders in adolescents: what can health care providers do? Journal of Adolescent Health. 2009; 44:206-213.

7. Campos P, Saguy A, Ernsberger P, et al. The epidemiology of overweight and obesity: public health crisis or moral panic? International Journal of Epidemiology. 2005; 35: 55-60

8. Rich E, Evans J. 'Fat Ethics'- The obesity discourse and body politics. Social Theory \& Health. 2005; 3(34):341-358.

9. Wildman RP, Muntner P, Reynolds $\mathrm{K}$, et al. The obese without cardiometabolic risk factor clustering and the normal weight with cardiometabolic risk factors clustering: Prevalence and correlates of 2 phenotypes among the US population (NHANES 1999-2004). Archives of Internal Medicine. 2008;168(15):1617-1624.

10. Oprana HM, Berthelot J-M, Kaplan MS, et al. BMI and Mortality: Results From a National Longitudinal Study of Canadian Adults. Obesity. 2010;18(1):214-218.

11. Bacon L, Keim N, Van Loan M, et al. Evaluating a "Non-diet" Wellness Intervention for Improvement of Metabolic Fitness, Psychological WellBeing and Eating and Activity Behaviors. International Journal of Obesity. 2002;26:854-865.

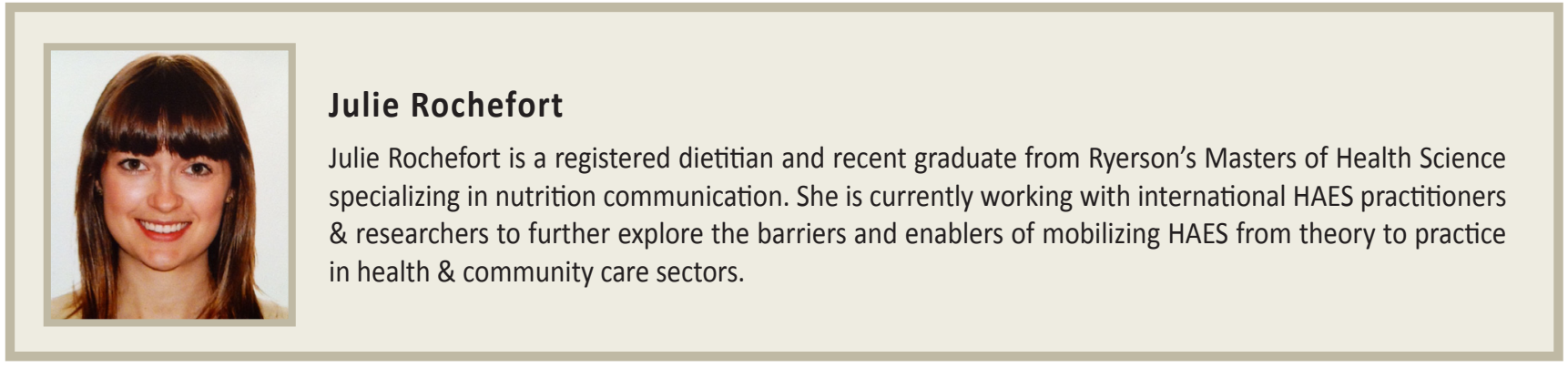

